\section{EZH2 does it}

\section{By Joanne Kotz, Senior Editor}

Because of the link between overexpression of the histone-methylating enzyme EZH2 and cancer, many researchers were surprised by a February paper that showed mutations in the histone methyltransferase actually inactivated the enzyme in B cell lymphomas. Now, Epizyme Inc. has painted a more complete picture by showing that these EZH2 mutations also can lead to an increase in activity. ${ }^{1}$

With small molecule inhibitors of EZH2 (enhancer of zeste homolog 2) in hand, Epizyme will now put its findings to the test in mouse models of lymphoma and other EZH2associated cancers.

EZH2 adds methyl groups to lysine 27 of histone 3, a position dubbed $\mathrm{H} 3 \mathrm{~K} 27$, in cells. The enzyme can add three sequential methyl groups, leading to mono-, di- or trimethylation of H3K27. Overexpression of EZH2 leads to increased trimethylation of $\mathrm{H} 3 \mathrm{~K} 27$, a modification that has been linked to a range of cancers including breast, prostate and lymphomas. ${ }^{2}$

In the February paper in Nature Genetics, researchers at the BC Cancer Agency and The University of British Columbia and colleagues reported that heterozygous mutations in EZH2 occurred in 7\%-22\% of patients with diffuse large B cell lymphoma (DLBCL) depending on disease subtype. All of the mutations occurred at a single tyrosine (Tyr641) in the enzyme.

The surprise came when biochemical assays showed that the EZH2 mutants had lost the ability to methylate a peptide substrate compared with the wild-type enzyme. ${ }^{3}$

Because an inactivating mutation in EZH2 ran contrary to the hypothesis that increased EZH2 expression correlated with cancer, Epizyme decided to investigate further.

Like the Canadian group, Epizyme found that Tyr641 mutants of EZH2 were much less active in taking an unmodified peptide substrate and adding the first methyl group.

Mutant and wild-type versions of the enzyme were similar in their ability to increase the methyl group number to two from one. However, the EZH2 mutants did a significantly better job than the wildtype enzyme at upping the peptide substrate's methyl group count from two to three. Thus, the mutant enzyme proved exceedingly good in vitro at creating the cancer-related trimethylated state.

Importantly, in cells in which the number of methyl groups on H3K27 was dynamically changing, this greater in vitro activity translated to an increase in methylation. Because the remaining copy of the wild-type enzyme was still able to catalyze the initial methylation, B cell lymphoma cell lines that were heterozygous for mutant EZH2 had greater $\mathrm{H} 3 \mathrm{~K} 27$ trimethylation than lymphoma cell lines that were homozygous for the wild-type enzyme.

Data were published in the Proceedings of the National Academy of Sciences. The Epizyme team was led by Robert Copeland, EVP and $\mathrm{CSO}$, and Victoria Richon, VP of biological sciences.

"The significance of these results is that it defines a clear and unambiguous direction for pursuing EZH2 inhibitors as targeted therapeutics for lymphoma patients with this mutation," said Copeland.

The February paper describing the Tyr641 mutations as leading to a loss of function "were hard to reconcile with the genetic evidence that the cancer cells always selected for mutations in the same residue (Tyr641), which usually is a characteristic of gain-of-function mutations," said Patrick Trojer, director of biology at Constellation

\section{Pharmaceuticals Inc.}

"The current work demonstrates that the Tyr641 mutation results in gain of function, which provides important information to the field and supports the plausible hypothesis that the EZH2 Tyr641 allele makes an oncogenic contribution to DLBCL."

Constellation has drug discovery programs targeting multiple classes of chromatin modifiers, including histone methyltransferases. The company has not disclosed specific targets.

The new data "set the record straight," said Kristian Helin, director of the Biotech Research \& Innovation Centre at the University of Copenhagen.

\section{Target validation}

The next outstanding question is whether inhibiting EZH2 will show efficacy in preclinical cancer models.

"The possibility of testing EZH2-targeted therapy in DLBCL is quite intriguing and could be a promising experimental therapy," said Ari Melnick, associate professor of medicine at Weill Cornell Medical College.

However, he noted that there are no published data yet demonstrating that mutant EZH2 drives oncogenesis in vivo.

"There is very strong evidence suggesting EZH2 is a good target in many cancers but there is no definitive proof," said Helin.

"Significant EZH2 overexpression with concomitant increases in $\mathrm{H} 3 \mathrm{~K} 27$ trimethylation have been reported in numerous cancer types, for instance, metastatic prostate cancer, hormone-refractory breast cancer and multiple myeloma," said Trojer. "But the causal link between EZH2 mutation, elevated H3K27 trimethylation levels and lymphoma cell growth has not yet been established."

The key experiment, according to both Helin and Trojer, will be to show efficacy in an in vivo model with either conditional knockout of EZH2 or small molecule inhibitors of the enzyme.

Epizyme soon may be in a position to do this experiment, according to Copeland. The company has identified potent small molecule inhibitors "with a range of selectivity for mutant EZH2 versus the wild-type enzyme," he told SciBX. 


\section{ANALYSIS}

\section{TARGETS \& MECHANISMS}

In cell culture studies, these inhibitors selectively kill patientderived lymphoma cells with EZH2 mutations, said Copeland. "We are encouraged by the pharmacological characteristics of the EZH2 inhibitor series," he added.

Based on these results, said Copeland, "we are currently gearing up to do our first in vivo efficacy experiments." The company plans to test EZH2 inhibitors in mouse xenograft models of human lymphoma as well as in additional mouse models of EZH2-related cancers.

Epizyme has filed and is filing patent applications covering EZH2 and related diagnostics and therapeutics. Copeland told SciBX that the work is currently not partnered.

Kotz, J. SciBX 3(47); doi:10.1038/scibx.2010.1401

Published online Dec. 9, 2010
REFERENCES

1. Sneeringer, C.J. et al. Proc. Natl. Acad. Sci. USA; published online Nov. 15, 2010; doi:10.1073/pnas.1012525107

Contact: Robert A. Copeland, Epizyme Inc., Cambridge, Mass. e-mail: RCopeland@epizyme.com

Contact: Victoria M. Richon, same affiliation as above e-mail: VRichon@epizyme.com

2. Copeland, R.A. et al. Nat. Rev. Drug Discov. 8, 724-732 (2009)

3. Morin, R.D. et al. Nat. Genet. 42, 181-185 (2010)

COMPANIES AND INSTITUTIONS MENTIONED

BC Cancer Agency, Vancouver, British Columbia, Canada

Constellation Pharmaceuticals Inc., Cambridge, Mass.

Epizyme Inc., Cambridge, Mass.

The University of British Columbia, Vancouver, British Columbia, Canada University of Copenhagen, Copenhagen, Denmark

Weill Cornell Medical College, New York, N.Y. 